\title{
Effect of N-terminal region of human parvovirus B19-VP1 unique region on cardiac injury in naïve mice
}

\author{
KUO-CHUAN HUNG ${ }^{1}$, ZI-YUN HUANG ${ }^{2}$, JIA LE YOW ${ }^{2,3}$, TSAI-CHING HSU ${ }^{2,4,5}$ and BOR-SHOW TZANG ${ }^{2-5}$ \\ ${ }^{1}$ Department of Anesthesiology, Chi Mei Medical Center, Tainan $710 ;{ }^{2}$ Institute of Medicine, \\ College of Medicine; ${ }^{3}$ Department of Biochemistry, School of Medicine, College of Medicine; \\ ${ }^{4}$ Immunology Research Center, Chung Shan Medical University; ${ }^{5}$ Department of Clinical Laboratory, \\ Chung Shan Medical University Hospital, Taichung 402, Taiwan
}

Received March 18, 2021; Accepted June 28, 2021

DOI: $10.3892 / \mathrm{mmr} .2021 .12399$

\begin{abstract}
A unique region of human parvovirus B19 virus-VP1 (B19V-VP1u) has been linked to a variety of cardiac disorders. However, the precise role of B19V-VPlu in inducing cardiac injury remains unknown. The present study investigated the effects of B19V-VP1u and different regions of B19V-VP1u, including B19V-VP1uA (residues 1-60), B19V-VP1uB (residues 61-129), B19V-VP1uC (residues 130-195) and B19V-VP1uD (residues 196-227), on inducing cardiac injury in naïve mice by zymography, immunoblotting, H\&E staining and cytokine immunoassay. A significantly higher MMP-9/MMP-2 ratio and increased levels of inflammatory cytokines, including IL- 6 and IL-1 $\beta$, were detected in the left ventricles of the mice injected with B19V-non-structural protein 1 (B19V-NS1) and B19V-VP1u, accompanied by increased expression levels of phosphorylated (p-)ERK and p-P38. Significantly upregulated expression levels of atrial natriuretic peptide (ANP), heart-type fatty acid-binding protein (H-FABP) and creatine kinase isoenzyme-MB (CK-MB), which are well-known cardiac injury markers, as well as increased infiltration of lymphocytes, were detected in the left ventricles of the mice injected with B19V-VP1, B19V-NS1 and B19V-VP1u. Moreover, a significantly higher MMP-9/MMP-2 ratio and increased levels of IL- 6 and IL-1 $\beta$ were observed in the left ventricles of the mice injected with B19V-VP1u, B19V-VP1u-A, B19V-VP1u-B and B19V-VP1u-C, accompanied by upregulated p-ERK
\end{abstract}

Correspondence to: Dr Bor-Show Tzang, Department of Biochemistry, School of Medicine, College of Medicine, Chung Shan Medical University, 110, Sector 1, Jianguo North Road, Taichung 402, Taiwan

E-mail: bstzang@csmu.edu.tw

Dr Tsai-Ching Hsu, Institute of Medicine, College of Medicine, Chung Shan Medical University, 110, Sector 1, Jianguo North Road, Taichung 402, Taiwan

E-mail: htc@csmu.edu.tw

Key words: human parvovirus B19 virus, B19 virus-VP1 unique region, cardiac injury and p-P38 expression. Notably, significantly lower levels of IL-6 and IL-1 $\beta$ were observed in the left ventricles of the mice injected with B19V-VP1uD. Furthermore, significantly increased ANP, H-FABP and CK-MB expression levels were detected in the left ventricles of the mice injected with B19V-VP1u, B19V-VP1u-A and B19V-VP1u-B, along with enhanced infiltration of lymphocytes. Significantly higher serum IL-1 $\beta$, IL-6, TNF- $\alpha$ and IFN- $\gamma$ levels were also detected in the mice injected with B19V-VP1u, B19V-VP1u-A and B19V-VP1u-B. To the best of our knowledge, the findings of the present study were the first to demonstrate that the $\mathrm{N}$-terminal region (residues 1-129) of B19V-VP1u induces an increase in the levels of cardiac injury markers, thus providing evidence for understanding the possible functional regions within B19V-VP1u.

\section{Introduction}

B19 virus (B19V), known as a human parvovirus, is a widespread DNA virus that may be found in patients across a wide age range (0-94 years); the immunity to $\mathrm{B} 19 \mathrm{~V}$ was reported to be $\sim 78 \%$ in individuals $>50$ years old in various countries between 1992 and 1998 (1). The B19V genome encodes a non-structural protein (NS1), two capsid proteins (VP1 and VP2) and two smaller proteins, 7.5 and $11 \mathrm{kDa}$ in weight (1). Of the $\mathrm{B} 19 \mathrm{~V}$ capsid proteins, $\sim 95 \%$ are VP2, and the remaining $5 \%$ are VP1. The 227 amino acids at the $\mathrm{N}$-terminal region of B19V-VP1u are known as the B19V-VP1-unique region (VP1u), which shares the same amino acid sequence with B19V-VP1 (2). B19V-NS1 is known to possess enzymatic activities, including helicase and ATPase activities, and DNA-binding activities that regulate viral transcription and replication $(3,4)$. B19V-NS1 also increases the expression of IL-6 and TNF- $\alpha$ in host cells and induces cell apoptosis $(5,6)$. The major capsid protein of B19V, VP2, can bind P antigen (globoside) on the cell surface for viral attachment, and the minor capsid protein VP1 is associated with the induction of immune responses during infection (7-9).

$\mathrm{B} 19 \mathrm{~V}$ infection has been linked to a range of clinical manifestations, such as myocarditis $(10,11)$, hepatitis (12), nephrotic syndrome (13), thyroid disease (14), nerve damage (15), several types of cancer (16) and autoimmune diseases (17). To 
determine the association between $\mathrm{B} 19 \mathrm{~V}$ infection and cardiac disorders, the study of the presence of the B19V genome in cardiac tissues has attracted increased attention. Indeed, various studies have reported the presence of the B19V genome in endomyocardial tissues $(18,19)$. Similar results were also obtained from the myocardium of patients with dilated cardiomyopathy (DCM) $(20,21)$, fulminant myocarditis, sudden heart failure or chronic inflammatory cardiomyopathy $(22,23)$.

B19V-VP1u has been reported to significantly increase TNF- $\alpha$ expression in H9c2 cells (24). Accumulating evidence has indicated that immunization of $\mathrm{BALB} / \mathrm{c}$ mice with B19V-VP1u proteins can cause DCM (11). Moreover, the administration of B19V-VP1u in naïve mice can elicit ultrastructural changes of the heart and significantly increase the levels of various markers of cardiac injury, such as lactate dehydrogenase, creatine kinase isoenzyme and $\alpha$-hydroxybutyric acid dehydrogenase (25). Significantly aggravated inflammation, including elevated MMP-9 activity and levels of inflammatory cytokines (IL-1 $\beta$, IL- 6 and TNF- $\alpha$ ), has been detected in the left ventricles of the hearts of NZB/W F1 mice receiving B19V-VP1u IgG (26). Similar findings were also observed in patients with systemic lupus erythematosus with DCM, who possessed antibodies against B19V-VP1u, indicating an association between B19V-VP1u and DCM (27). These studies strongly suggest a pathological role of B19V-V1u in cardiomyopathy. Notably, our previous studies revealed the differential effects of truncated B19V-VP1u fragments on autoimmunity, particularly anti-phospholipid syndrome $(28,29)$. However, the antigenicity of B19V-VP1u in causing cardiac damage remains unknown. Therefore, the aim of the present study was to further elucidate the roles of different B19V-VP1u fragments in inducing cardiac injury.

\section{Materials and methods}

Human parvovirus B19V proteins. Human B19V recombinant proteins were prepared as described in our previous publications $(28,29)$. The B19V-NS1 protein was purified using the Profinia protein purification system (Bio-Rad Laboratories, Inc.) (28). The DNA sequences encoding B19-VP1, VP1u and truncated B19V-VP1uA (residues 1-60), B (residues 61-129), C (residues 130-195) and D (residues 196-227) were ligated into a pET-32a vector-Novagene (MilliporeSigma). These recombinant parvovirus B19 proteins were further induced with 1 $\mathrm{mM}$ IPTG for $3 \mathrm{~h}$ at $37^{\circ} \mathrm{C}$ and purified with a PureProteome $\mathrm{TM}^{\mathrm{TM}}$ Nickel Magnetic Beads system (MilliporeSigma). The purified proteins were further analyzed using high performance liquid chromatography (Waters HPLC 600 System; Waters Corporation). Briefly, $400 \mu \mathrm{l}(0.2 \mathrm{mg} / \mathrm{ml})$ purified proteins were injected into a $13-\mu$ m column (Superdex ${ }^{\circledR} 75$ 10/300 GL; MilliporeSigma) and eluted with elution buffer $(25 \mathrm{mM}$ Tris-HCL, pH 8.0; $150 \mathrm{mM} \mathrm{NaCl}$ ) at a flow rate of $0.5 \mathrm{ml} / \mathrm{min}$. The purity of the purified proteins ranged between 98.1 and $99.3 \%$, and the endotoxin levels of purified recombinant proteins were all under the limits of detection ( 0.25 endotoxin units/ml).

Ethics and animal. Animal experiments were performed in accordance with the principles of replacement, refinement and reduction and were approved by the Institutional
Animal Care and Use Committee (IACUC) of Chung Shan Medical University, Taichung (approval no. 1676; approved on December 2015). A total of 32 BALB/c BYJNarl female mice (age, 7 weeks; weight, 19-21 g) were obtained from the National Laboratory Animal Center and administrated under the IACUC at Chung Shan Medical University (Taichung, Taiwan). All animals were maintained in an air-conditioned room with a 12 -h light-dark cycle at $22^{\circ} \mathrm{C}$, and the relative humidity in the room was $55 \%$. Animals were allowed free access to water and standard laboratory chow (Lab Diet 5001; PMI Nutrition International Inc.).

After 1 week of acclimation, the 8-week-old animals were randomly divided into eight groups ( $\mathrm{n}=4$ per group) as follows: i) Control group (PBS); ii) B19V-VP1 group; iii) B19V-NS1 group; iv) B19V-VP1u group; v) VP1u-A group; vi) VP1u-B group; vii) VP1u-C group; and viii) VP1u-D group. As described in previous reports $(30,31)$ and in our previous publication (29), the initial and booster injections (2-3 times) are necessary to obtain optimal antibody responses. A total of $20 \mu \mathrm{g}$ purified recombinant proteins or PBS was mixed 1:1 (v/v) with Freund's complete adjuvant (MillporeSigma), and subcutaneously injected into mice of each group on day 1 . The mice were then boosted with $20 \mu \mathrm{g}$ recombinant proteins or PBS mixed 1:1 (v/v) with Freund's incomplete adjuvant (MillporeSigma) twice times each on days 14 and 28. All animals were fasted for $12 \mathrm{~h}$ before sacrifice with $\mathrm{CO}_{2}$ when they reached 16-weeks of age. A displacement rate of $20 \%$ of the chamber volume with $\mathrm{CO}_{2}$ per min was used to euthanize the mice (performed in April 2018). The blood from the heart and the left ventricle tissues of mice were harvested (at 10 a.m.) and stored at $-80^{\circ} \mathrm{C}$ until required for subsequent analysis. To confirm the induction of immunity by B19V-V1u and truncated B19V-VP1u recombinant proteins, absorption experiments were performed using a competitive (comp) ELISA (29) to verify the specificity of the antibodies in naïve mice receiving B19V-VP1u or truncated B19V-VP1u recombinant proteins (Fig. S1). Whole blood samples were obtained from the hearts of mice immunized with B19-VP1u or truncated B19V-VPlu recombinant proteins as aforementioned and were centrifuged at $1,500 \mathrm{x}$ for $10 \mathrm{~min}$ at room temperature to obtain the heart sera. For the absorption experiments (comp ELISA), the sera were pre-incubated with $500 \mu \mathrm{M}$ VP1u or truncated VPlu proteins for $1 \mathrm{~h}$ at $37^{\circ} \mathrm{C}$. Subsequently, all anti-sera (non-comp or comp groups) against $2 \mu \mathrm{g}$ B19-VP1u or truncated B19V-VPlu recombinant proteins were immobilized on the surface of each sample well of 96-well plates. After incubation at room temperature for $120 \mathrm{~min}$, the liquid from each sample well was removed, and the wells were washed with PBS and subsequently incubated with horseradish peroxidase (HRP)-conjugated rabbit anti-mouse $\mathrm{IgG}$ (cat. no. A9044; MilliporeSigma) at a dilution of 1:1,000. After incubation at room temperature for $60 \mathrm{~min}$, the liquid was removed from each sample well and the wells were washed with PBS. The color reaction was performed using $1 \mathrm{mg} / \mathrm{ml}$ substrate 2,2'azino-di-(3-ethylbenzthiazolin-6-sulphonic acid) (MilliporeSigma) in the presence of $0.005 \% \mathrm{H}_{2} \mathrm{O}_{2}$ at room temperature for $15 \mathrm{~min}$. A cutoff value was determined using sera from CTL mice, and the value was regarded as positive. Notably, the non-'comp' groups had enhanced absorbance as inoculation raised an immune response, whereas the 'comp' 

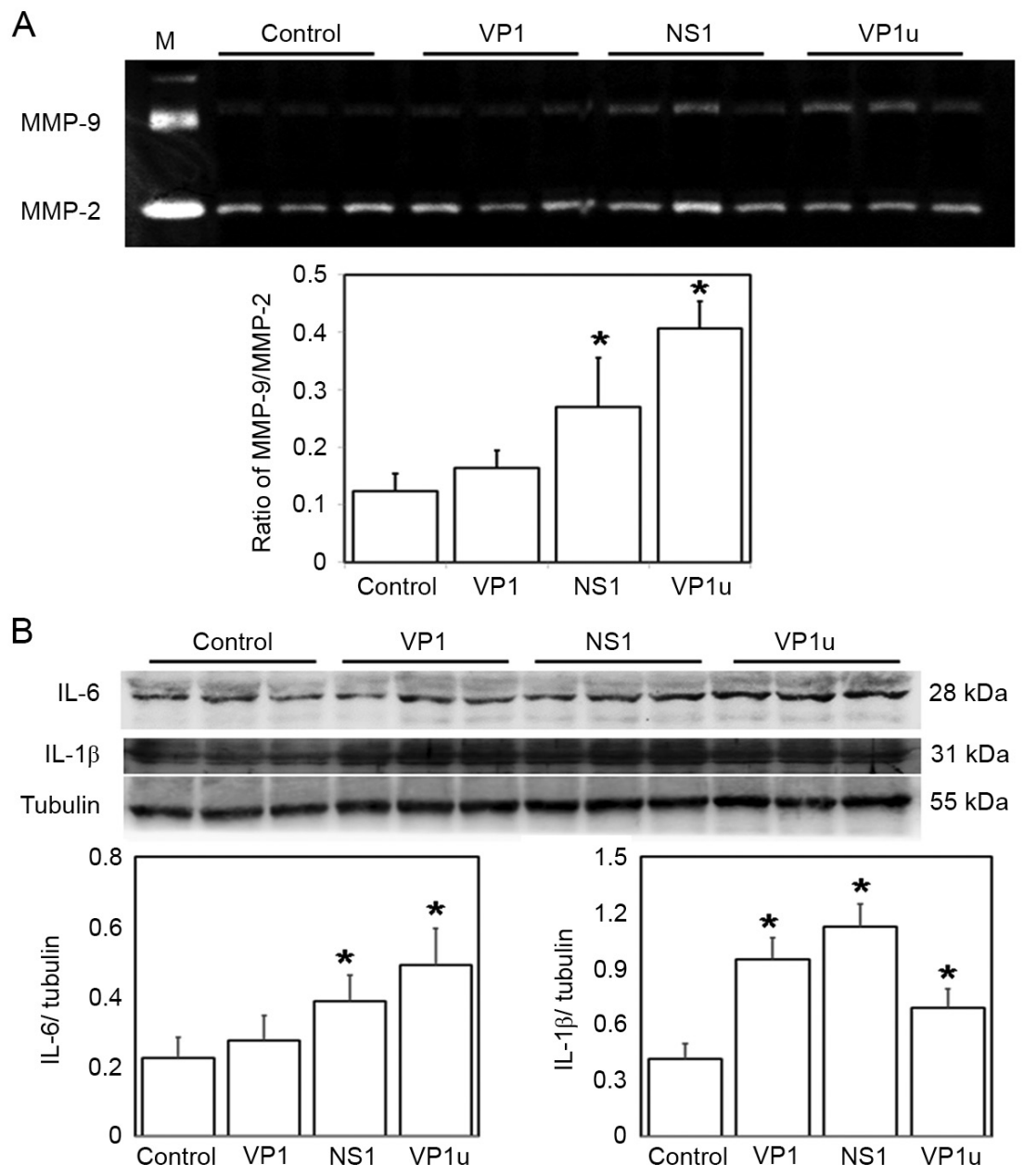

Figure 1. Effects of B19V-NS1, VP1 and VP1u on MMP-9 and MMP-2 activities and IL-6 and IL-1 $\beta$ expressions. (A) MMP-9 and MMP-2 activities in left ventricle tissues of the mice receiving different treatments (PBS, VP1, NS1 or VP1u). The activity ratio of MMP-9/MMP-2 is shown in the lower panel. M indicates mouse serum and was used as a positive control. (B) Protein expression levels of IL- 6 and IL-1 $\beta$ in left ventricle tissues of the mice receiving different treatments. The lower panel shows the relative levels of IL- 6 and IL-1 $\beta$ based on tubulin amount. Similar results were obtained in three-repeated experiments. ${ }^{*} \mathrm{P}<0.05$ vs. Control (mice treated with PBS). $\mathrm{n}=4$ for each group. B19V, B19 virus; B19V-NS1, B19V-non-structural protein 1; VP1u, VP1-unique region.

groups had reduced absorbance as antibodies were bound to the proteins from the initial absorption experiment (Fig. S1).

Gel zymography. MMP-9 and MMP-2 activities were measured as described previously (32). Protein concentrations were determined using a Bradford assay (Bio-Rad Laboratories, Inc.) and quantified using a U3000 spectrophotometer (Hitachi Ltd.). A total of $25 \mu \mathrm{g}$ left ventricular tissue of mice from each experimental group was resolved on an $8 \%$ SDS-gel, via SDS-PAGE, that contained $0.1 \%$ gelatin. MMP-9 and MMP-2 activities were detected by staining the gel with $0.5 \%$ coomassie brilliant blue R-250 for $1 \mathrm{~h}$ at room temperature after soaking in reaction solution (40 mM Tris- $\mathrm{HCl}, 10 \mathrm{mM}$ $\mathrm{CaCl}_{2}$ and $0.02 \% \mathrm{NaN}_{3}$ ) for $18 \mathrm{~h}$. A gel densitometry system (Alpha-Imager 2200; ProteinSimple) was used to quantify the relative MMP levels.

Protein preparation and immunoblotting. Protein concentration was determined by a modified Bradford's assay using a U3000 spectrophotometer at $595 \mathrm{~nm}$ with BSA (MilliporeSigma) as the standard. A total of $15 \mu \mathrm{g}$ left ventricular tissue from each animal was resected and homogenized in
$600 \mu 1$ lysis buffer (PRO-PREP ${ }^{\mathrm{TM}}$; Intron Biotechnology, Inc.) with a tissue homogenizer (Polytron RT 3000; Brinkmann Instruments, Inc.). The homogenized tissues were then placed on ice for $30 \mathrm{~min}$, and the supernatants of tissue lysates were harvested by centrifuging at $4^{\circ} \mathrm{C}$ at $18,928 \times \mathrm{g}$ for $15 \mathrm{~min}$. For immunoblotting, extracted proteins (25 $\mu \mathrm{g} /$ lane) were separated using 12 or $15 \%$ SDS-gels, and transferred to nitrocellulose membranes (Bio-Rad Laboratories, Inc.). After blocking in $5 \%$ non-fat dry milk for $1 \mathrm{~h}$ at $25^{\circ} \mathrm{C}$, membranes were incubated with antibodies against IL-6 (1:500; cat. no. sc-1265), IL-1 $\beta$ (1:500; cat. no. sc-7884), ERK (1:1,000; cat. no. sc-514302), P38 (1:1,000; cat. no. sc-7972), phosphorylated (p)-P38 (1:1,000; cat. no. sc-7973), atrial natriuretic peptide (ANP; 1:500; cat. no. sc-20158) (all from Santa Cruz Biotechnology, Inc.), heart-type fatty acid-binding protein (H-FABP; 1:500; cat. no. ab16915), creatine kinase-MB (CK-MB; 1:500; cat. no. ab71722 (both from Abcam), p-ERK (1:1,000; cat. no. 05-797R; MilliporeSigma) and $\alpha$-tubulin (1:500; cat. no. PA5-16891; Thermo Fisher Scientific, Inc.) for $3 \mathrm{~h}$ at $25^{\circ} \mathrm{C}$. The membranes were subsequently incubated with HRP-conjugated secondary antibodies (1:5,000; cat. nos. sc-2004 or sc-2005; Santa Cruz Biotechnology, Inc.) 


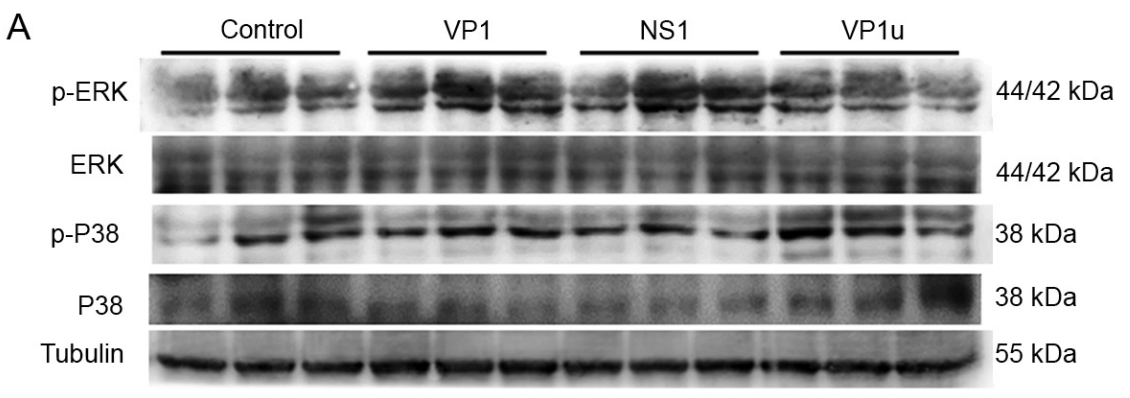

B
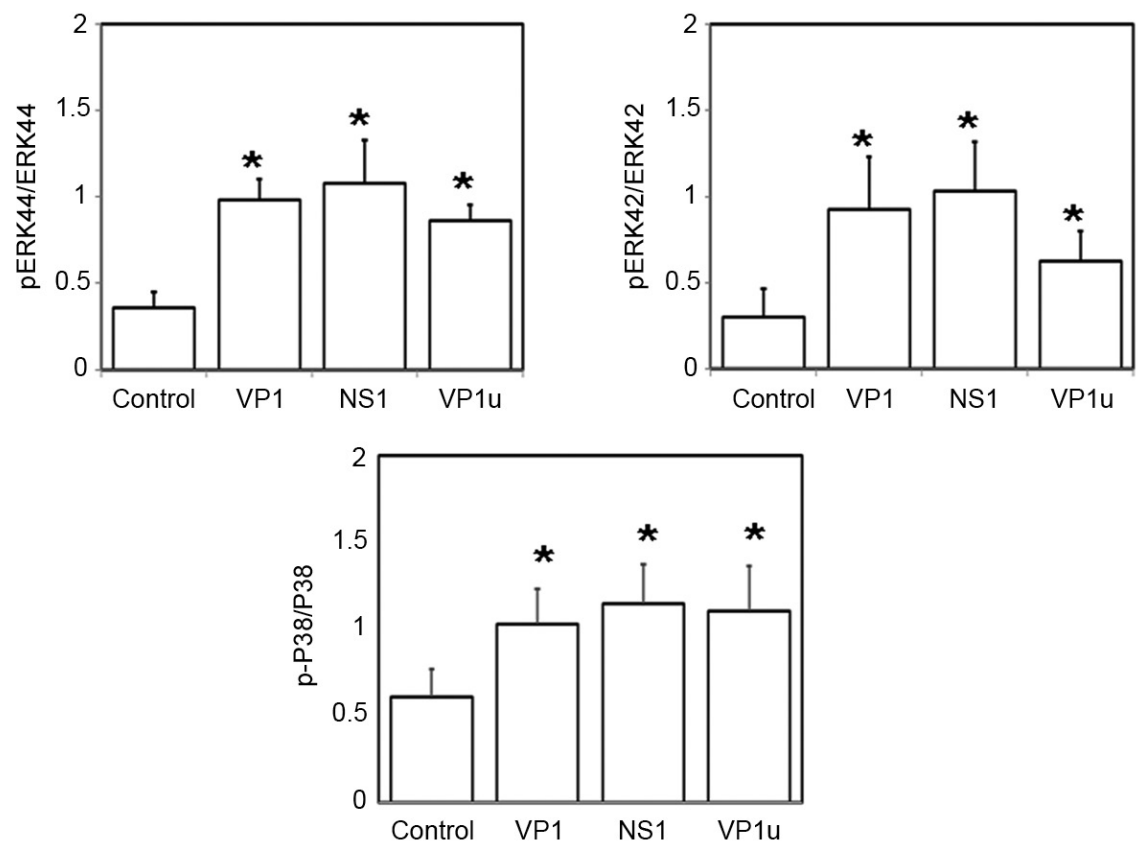

Figure 2. Effects of B19V-NS1, VP1 and VP1u on the phosphorylation of ERK and P38. (A) Protein expression levels of ERK, p-ERK, P38, and p-P38 in the left ventricle tissues of the mice receiving different treatments (PBS, VP1, NS1 or VP1u). (B) Relative levels of p-ERK and p-P38 are based on total protein expression. Similar results were obtained in three-repeated experiments. " $\mathrm{P}<0.05$ vs. Control (mice treated with PBS). $\mathrm{n}=4$ for each group. B19V, B19 virus; B19V-NS1, B19V-non-structural protein 1; VP1u, VP1-unique region; p-, phosphorylated.

at $25^{\circ} \mathrm{C}$ for $1.5 \mathrm{~h}$. Signals were visualized using Immobilon Western Chemiluminescent HRP Substrate (MilliporeSigma), and densitometry analysis was performed using an imaging analyzer(GEImageQuant TL 8.1;GEHealthcare Life Sciences).

$H \& E$ staining. For H\&E staining, the left ventricle tissues of mice were immersed in $10 \%$ formalin at $25^{\circ} \mathrm{C}$ for $24 \mathrm{~h}$ and embedded in paraffin. The tissue blocks were sliced into 4- $\mathrm{mm}$ sections, deparaffinized and dehydrated. Next, the slides were stained with hematoxylin and eosin after processing at $25^{\circ} \mathrm{C}$ for 5 min with 100, 95 and $75 \%$ ethanol. Each slide was subsequently immersed in $85 \%$ ethanol and $100 \%$ ethanol twice (20 min each immersion). Finally, the slides were immersed in xylene twice for $2 \mathrm{~min}$. Photomicrographs were observed using a light microscope (Zeiss Axiophot microscope; Zeiss AG) at x200 and x400 magnifications.

Mouse cytokine immunoassay. The serum IL-1 $\beta$, IL-6, TNF- $\alpha$ and IFN- $\gamma$ levels of mice from each experimental group were detected with Bio-Plex mouse cytokine 8-plex assay kit, according to manufacturer's instructions (cat. no. Z6000004CF; Bio-Rad Laboratories, Inc.) and Bio-Plex manager software version 3.0 using five parametric curve fitting (Bio-Rad Laboratories, Inc.).

Statistical analysis. GraphPad Prism 5 software (GraphPad Software, Inc.) was used to calculate the significant differences among groups using a one-way ANOVA followed by Tukey's multiple-comparisons test. All data are presented as the mean \pm SEM, and were verified in $\geq 3$ independent experiments. $\mathrm{P}<0.05$ was considered to indicate a statistically significant difference.

\section{Results}

Effects of different recombinant B19V proteins on inducing markers of cardiac inflammation in naïve mice. To determine the influence of different B19V proteins on increasing cardiac inflammation, MMP-9 and MMP-2 activities were detected in the left ventricles of mice treated with PBS (Control), or recombinant B19V-VP1, B19V-NS1 or B19V-VP1u proteins. A significantly higher MMP-9/MMP-2 ratio was observed in left ventricular tissues of the mice from the B19V-NS1 and B19-VP1u groups compared with the control group (Fig. 1A). 
A

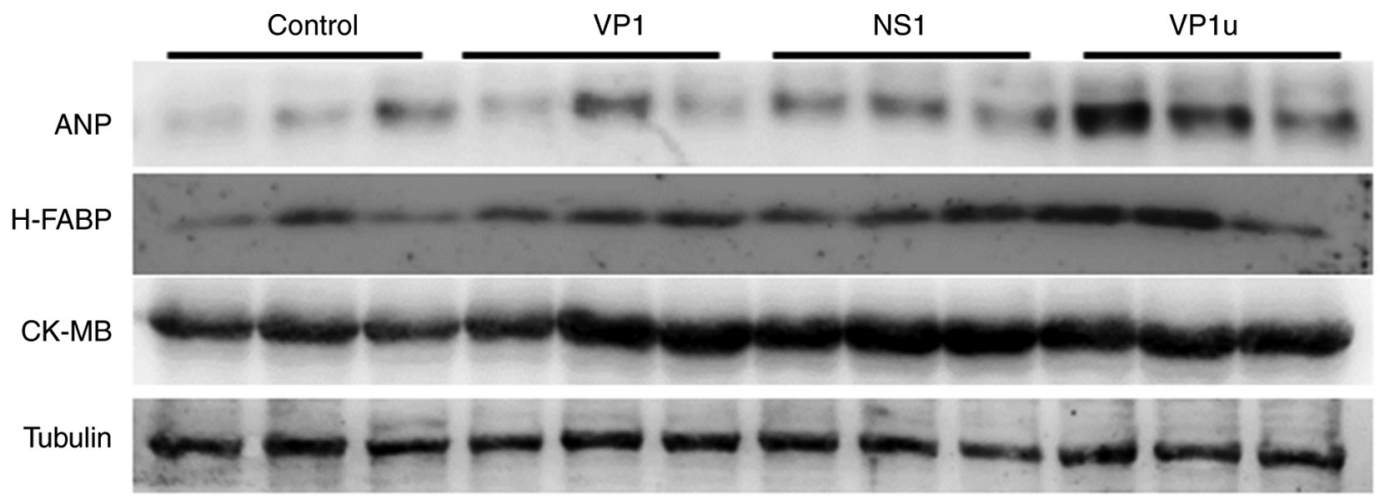

$17 \mathrm{kDa}$

$15 \mathrm{kDa}$

$43 \mathrm{kDa}$
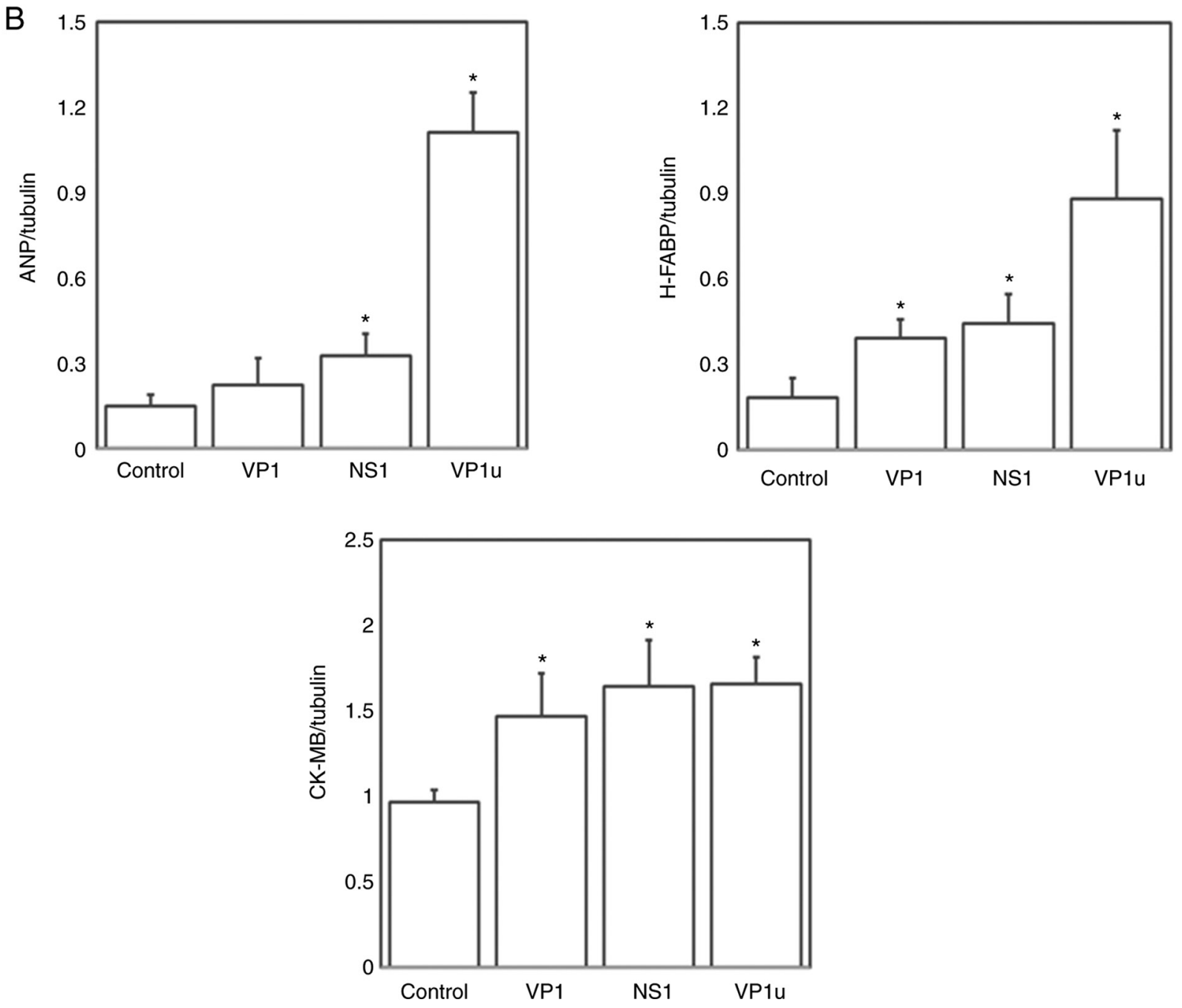

Figure 3. Effects of B19V-NS1, VP1 and VP1u on the expression levels of ANP, H-FABP and CK-MB. (A) Protein expression levels of ANP, H-FABP and CK-MB in the left ventricle tissues of the mice receiving different treatments (PBS, VP1, NS1 or VP1u). (B) Relative levels of ANP, H-FABP and CK-MB are based on tubulin expression. Similar results were obtained in three-repeated experiments. " $\mathrm{P}<0.05$ vs. Control (mice treated with PBS). $n=4$ for each group. B19V, B19 virus; B19V-NS1, B19V-non-structural protein 1; VP1u, VP1-unique region; ANP, atrial natriuretic peptide; H-FABP, heart-type fatty acid-binding protein; CK-MB, creatine kinase isoenzyme-MB.

The levels of the inflammatory cytokines, IL- 6 and IL-1 $\beta$, were also detected. Significantly higher amounts of IL-6 were observed in the left ventricular tissues of the mice from the B19V-NS1 and B19-VP1u groups compared with the control group (Fig. 1B). Moreover, significantly increased IL-1 $\beta$ levels were detected in left ventricular tissues of mice from the B19V-VP1, B19V-NS1 and B19-VP1u groups compared with the control group (Fig. 1B). In addition, significantly elevated p-ERK/ERK and p-P38/P38 ratios were observed in left ventricular tissues of the mice from the B19V-VP1, B19V-NS1 and B19-VP1u groups compared with the controls (Fig. 2).

Effects of different recombinant B19V proteins on inducing cardiac injury indicators in naïve mice. To understand the 


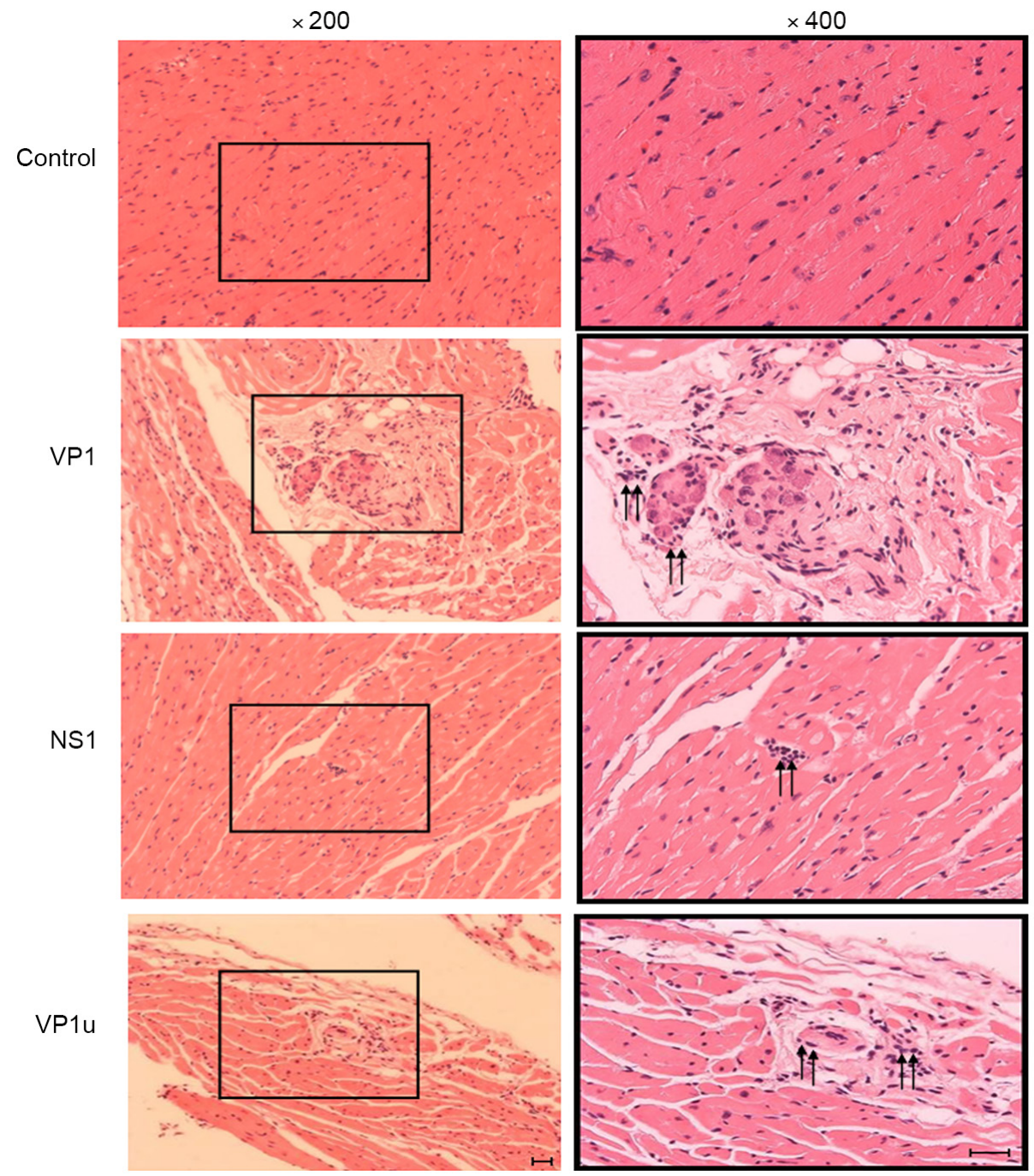

Figure 4. H\&E staining of the left ventricles in naïve mice treated with B19V-NS1, VP1 or VP1u. Lymphocyte infiltration is indicated by an arrow in left ventricle tissues of naïve mice receiving different treatments (PBS, VP1, NS1 or VP1u). The images in the left panel and right panel were magnified by x200 and x400, respectively. Scale bar, $10 \mu \mathrm{m}$. B19V, B19 virus; B19V-NS1, B19V-non-structural protein 1; VP1u, VP1-unique region.

influence of different B19V proteins on increasing cardiac injury in naïve mice, various indicators of myocardial injury, including ANP, H-FABP and CK-MB, were measured via immunoblotting. Significantly increased ANP expression was detected in the left ventricular tissues of mice from the B19V-NS1 and B19V-VP1u groups compared with the control group (Fig. 3). Moreover, significantly elevated H-FABP and CK-MB expression was observed in left ventricular tissues of mice from the B19V-VP1, B19V-NS1 and B19V-VP1u groups compared with the control group (Fig. 3). Accordingly, infiltration of lymphocytes was observed in the left ventricles of mice injected with B19V-VP1, B19V-NS1 and B19V-VP1u proteins (Fig. 4).

Effects of different regions of recombinant B19V-VPIu proteins on inducing increases in the levels of cardiac inflammatory markers in naïve mice. To further verify the effects of different regions of B19V-VP1u on cardiac inflammation, the MMP-9 and MMP-2 activities, as well as IL-6 and IL-1 $\beta$ levels were detected in the left ventricles of mice treated with PBS, or recombinant B19V-VP1u, B19V-VP1u-A,
B19V-VP1u-B, B19V-VP1u-C or B19V-VP1u-D proteins. A significantly higher MMP-9/MMP-2 ratio was detected in left ventricular tissues of mice from the B19V-VP1u, B19V-VP1u-A, B19V-VP1u-B and B19V-VP1u-C groups compared with the controls (Fig. 5A). Moreover, significantly higher levels of IL- 6 and IL-1 $\beta$ were detected in the left ventricular tissues of the mice from the B19V-VP1u, B19V-VP1u-A, B19V-VP1u-B and B19V-VP1u-C groups compared with the controls (Fig. 5B). Conversely, significantly lower IL-6 and IL- $1 \beta$ levels were observed in the left ventricular tissues of mice from the B19V-VP1u-D group compared with the controls (Fig. 5B). Moreover, significantly increased ratios of p-ERK/ERK and p-P38/P38 were observed in the left ventricular tissues of mice from the B19V-VP1u, B19V-VP1u-A, B19V-VP1u-B and B19V-VPlu-C groups compared with the controls (Fig. 6).

Effects of different regions of recombinant B19V-VPIu on indicators of cardiac injury in naïve mice. To investigate the effects of different regions of $\mathrm{B} 19 \mathrm{~V}$ proteins on increasing cardiac injury in naïve mice, the expression levels of ANP, H-FABP and CK-MB proteins were detected 
A
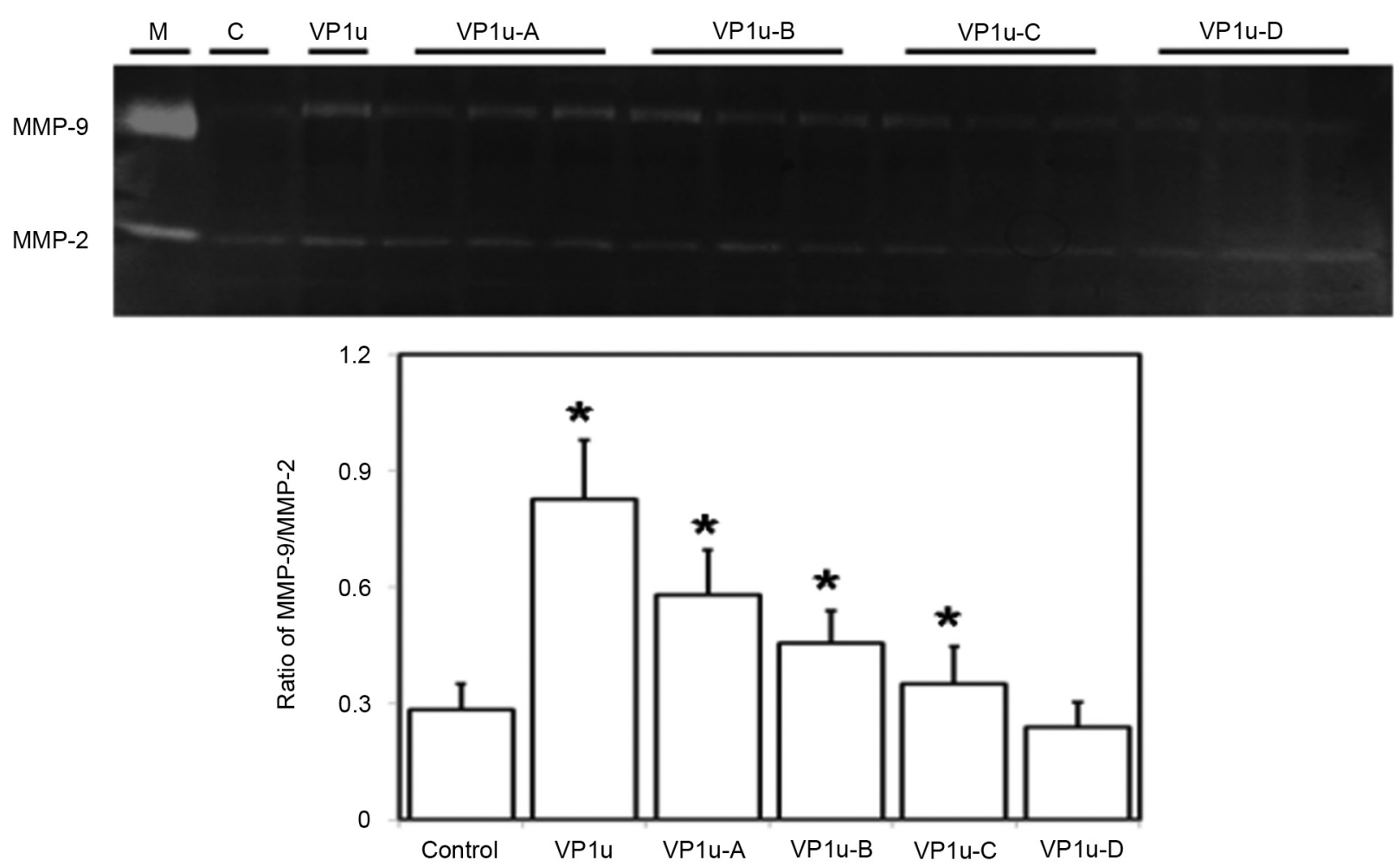

B
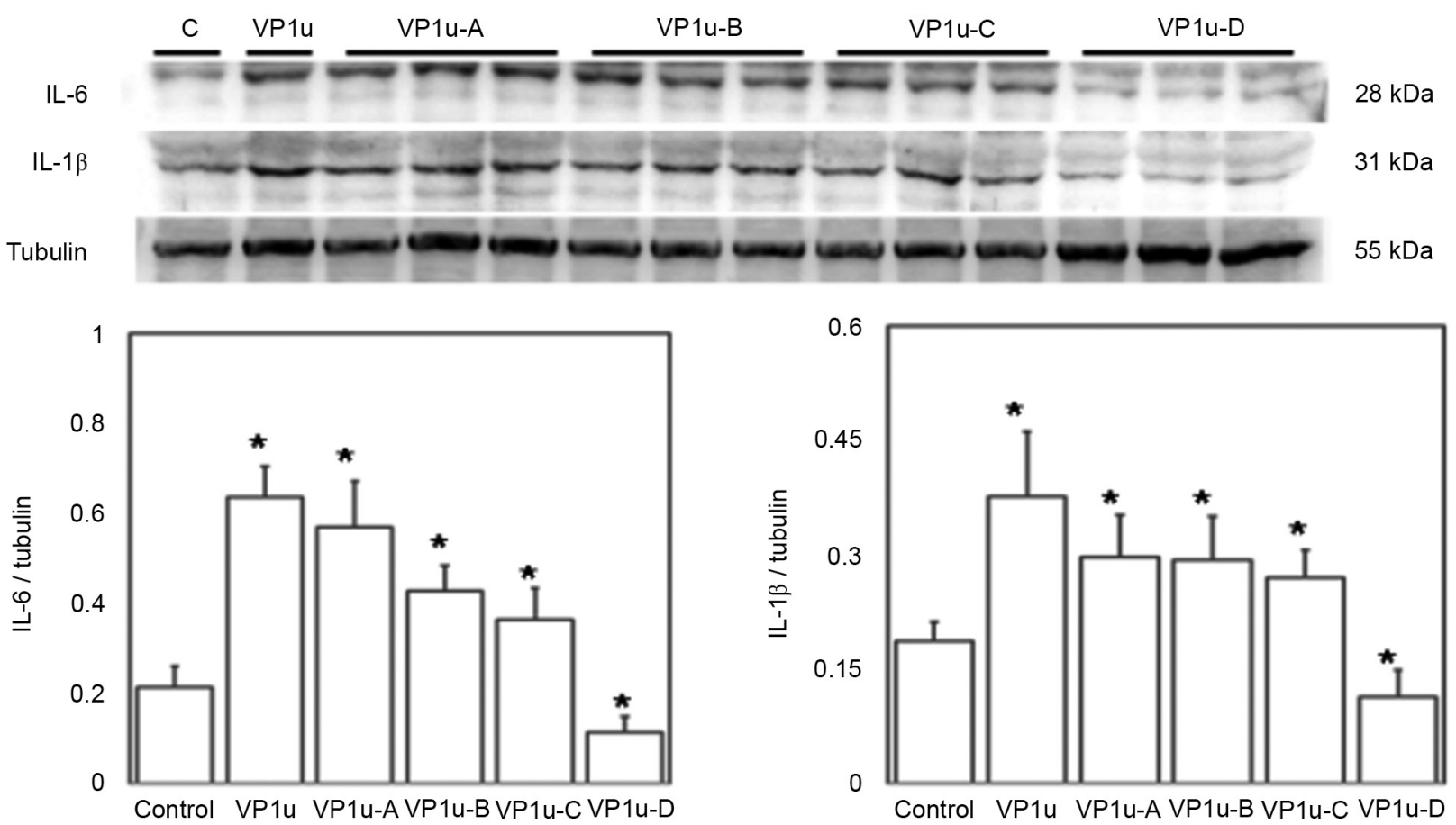

Figure 5. Effects of B19V-VPlu and its truncated fragments on MMP-9 and MMP-2 activities and IL-6 and IL-1 13 expression. (A) MMP-9 and MMP-2 activities in left ventricle tissues of mice receiving different treatments (PBS, VPlu, VPlu-A, VP1u-B, VP1u-C or VP1u-D). The MMP-9/MMP-2 activity ratio is shown in the lower panel. M indicates mouse serum and was used as a positive control; C indicates control mice treated with PBS. (B) Protein expression levels of IL- 6 and IL- $1 \beta$ in the left ventricle tissues of the mice receiving different treatments. The lower panel shows the levels of IL- 6 and IL- $1 \beta$ based on tubulin expression. Similar results were obtained in three-repeated experiments. ${ }^{\prime} \mathrm{P}<0.05$ vs. Control (mice treated with $\mathrm{PBS}$ ). $\mathrm{n}=4$ for each group. B19V, B19 virus; VP1u, VP1-unique region.

via immunoblotting. Significantly higher expression levels of ANP, H-FABP and CK-MB were observed in the left ventricles of mice injected with B19V-VP1u, B19V-VP1u-A and B19V-VP1u-B proteins compared with the controls (Fig. 7). Accordingly, increased infiltration of lymphocytes was observed in the left ventricles of mice injected with B19V-VP1u, B19V-VP1u-A, B19V-VP1u-B and B19V-VP1u-C (Fig. 8). In addition, significantly higher serum IL-1 $\beta$, IL-6, TNF- $\alpha$ and IFN- $\gamma$ levels were detected in mice injected with B19V-VP1u and B19V-VP1u-A, and only higher serum 
A

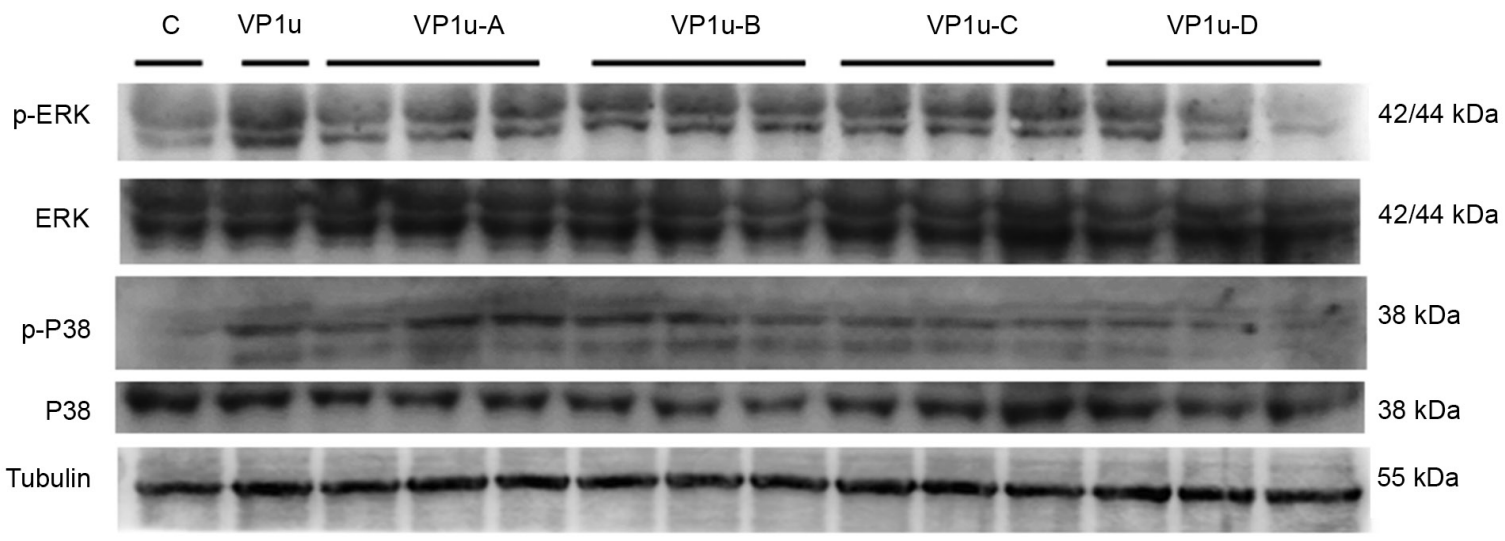

B
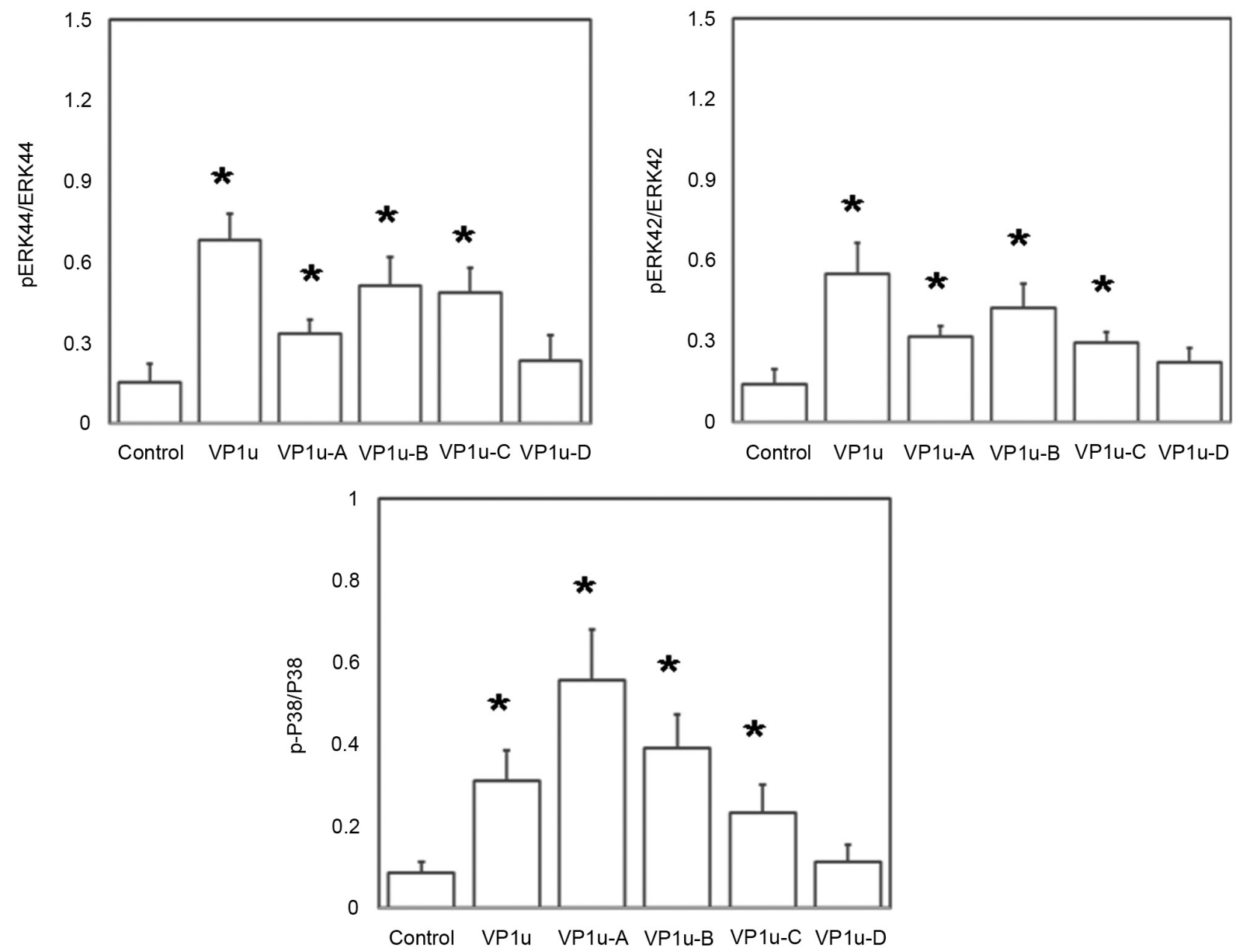

Figure 6. Effects of B19V-VP1u and its truncated fragments on the phosphorylation of ERK and P38. (A) ERK, p-ERK, P38 and p-P38 expression in left ventricle of the mice receiving different treatments (PBS, VP1u, VP1u-A, VP1u-B, VP1u-C or VP1u-D). C indicates control mice treated with PBS. (B) Relative expression levels of p-ERK and p-P38 are based on total protein expression. Similar results were obtained in three-repeated experiments. "P<0.05 vs. Control (mice treated with PBS). n=4 for each group. B19V, B19 virus; VP1u, VP1-unique region; p-, phosphorylated.

IL-1 $\beta$ and IL-6 levels were detected in mice injected with B19V-VP1u-B proteins compared with the controls (Fig. 9).

\section{Discussion}

Both B19V-NS1 and B19V-VPlu proteins have been linked to various heart disorders such as inflammatory cardiomyopathy and DCM (11,24-25). Notably, significantly increased cardiac inflammation was detected in NZB/W F1 mice receiving
B19V-VP1u IgG (26). In addition, among patients with systemic lupus erythematous, a potential association between B19V-VP1u and DCM was also reported (27). Although these findings revealed an association between B19V-VP1u and cardiac disorders, the roles of the antigenicity and functional motifs of B19V-VPlu in the induction of cardiac injury remain unknown. The present study identified a significantly increased MMP-9/MMP-2 ratio and elevated IL-6, IL-1 $\beta$, ANP, H-FABP and CK-MB levels in the left ventricles of mice 
A

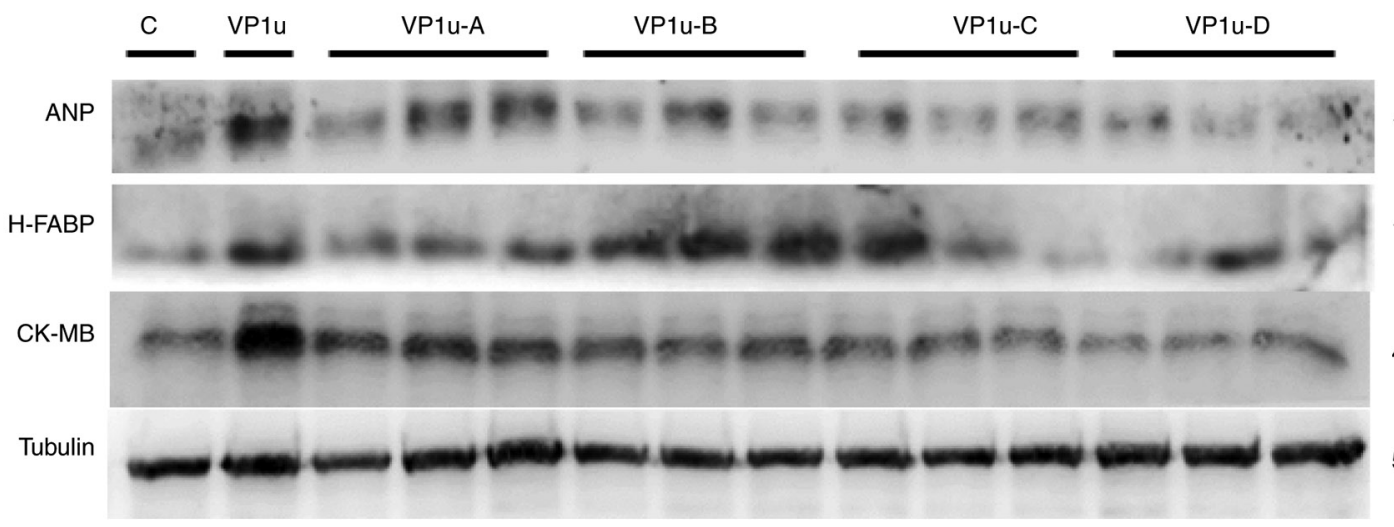

$17 \mathrm{kDa}$

$15 \mathrm{kDa}$

$43 \mathrm{kDa}$

$55 \mathrm{kDa}$

B
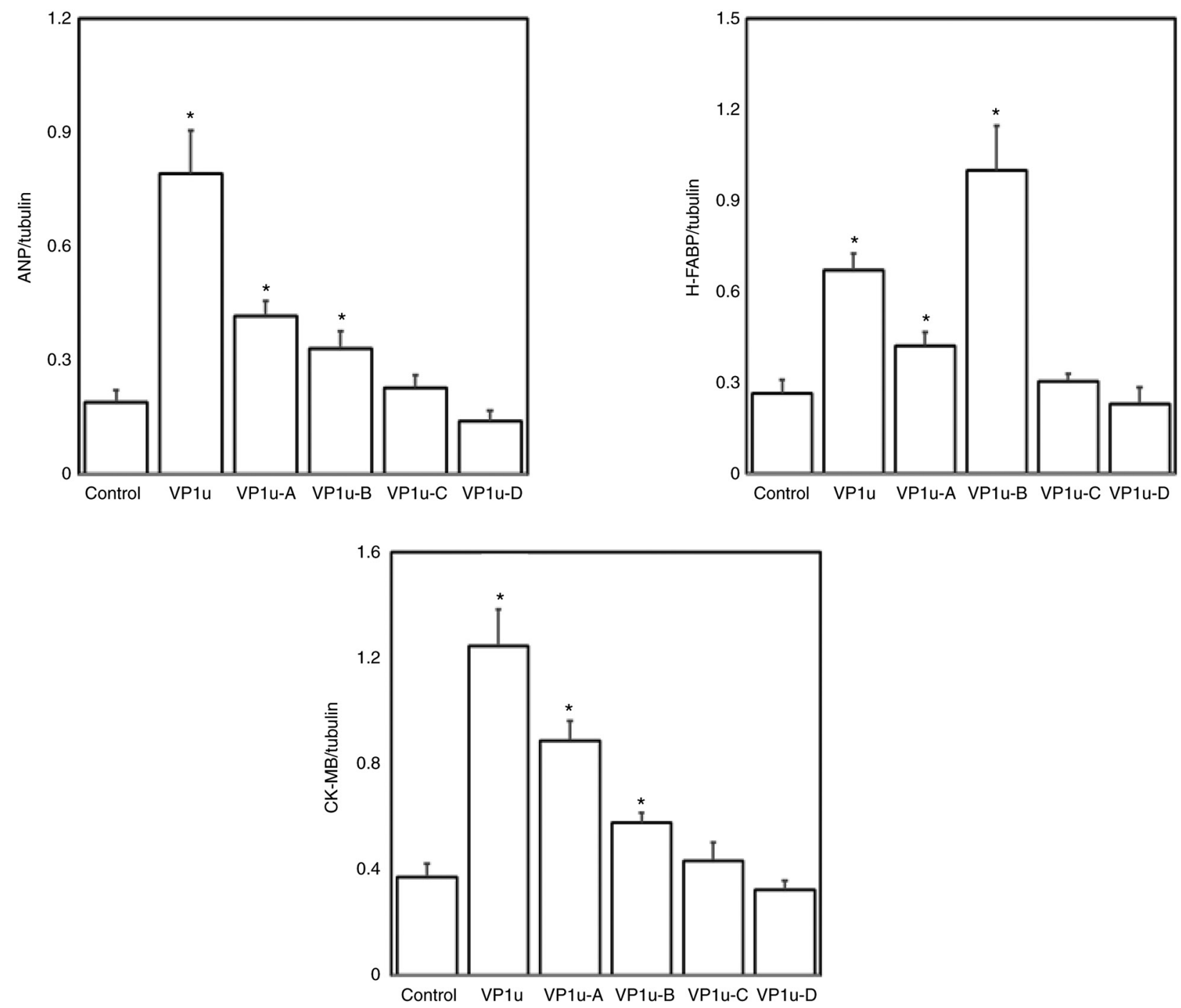

Figure 7. Effects of B19V-VPlu and its truncated fragments on expression levels of ANP, H-FABP and CK-MB. (A) Protein expression levels of ANP, H-FABP and CK-MB in the left ventricle tissues of the mice receiving different treatments (PBS, VPlu, VPlu-A, VPlu-B, VPlu-C or VPlu-D). C indicates control mice treated with PBS. (B) Relative levels of ANP, H-FABP and CK-MB are based on tubulin expression. Similar results were obtained in three-repeated experiments. " $\mathrm{P}<0.05$ vs. Control (mice treated with PBS). $\mathrm{n}=4$ for each group. B19V, B19 virus; VP1u, VP1-unique region; ANP, atrial natriuretic peptide; $\mathrm{H}-\mathrm{FABP}$, heart-type fatty acid-binding protein; CK-MB, creatine kinase isoenzyme-MB.

treated with B19V-NS1 and B19V-VP1u. Moreover, significantly increased MMP-9/MMP-2 ratios and IL-6 and IL-1 $\beta$ levels were detected in the left ventricles of mice treated with B19V-VP1u-A, B19V-VP1u-B and B19V-VP1u-C. Significantly higher levels of ANP, H-FABP and CK-MB proteins were also observed in the left ventricles of mice treated with B19V-VP1u, B19V-VP1u-A and B19V-VP1u-B, accompanied by significantly higher serum IL-1 $\beta$, IL- 6 , TNF- $\alpha$ and IFN- $\gamma$ levels. Conversely, significantly lower levels of IL-6 and IL-1 $\beta$ were observed in the left ventricles of mice treated with 

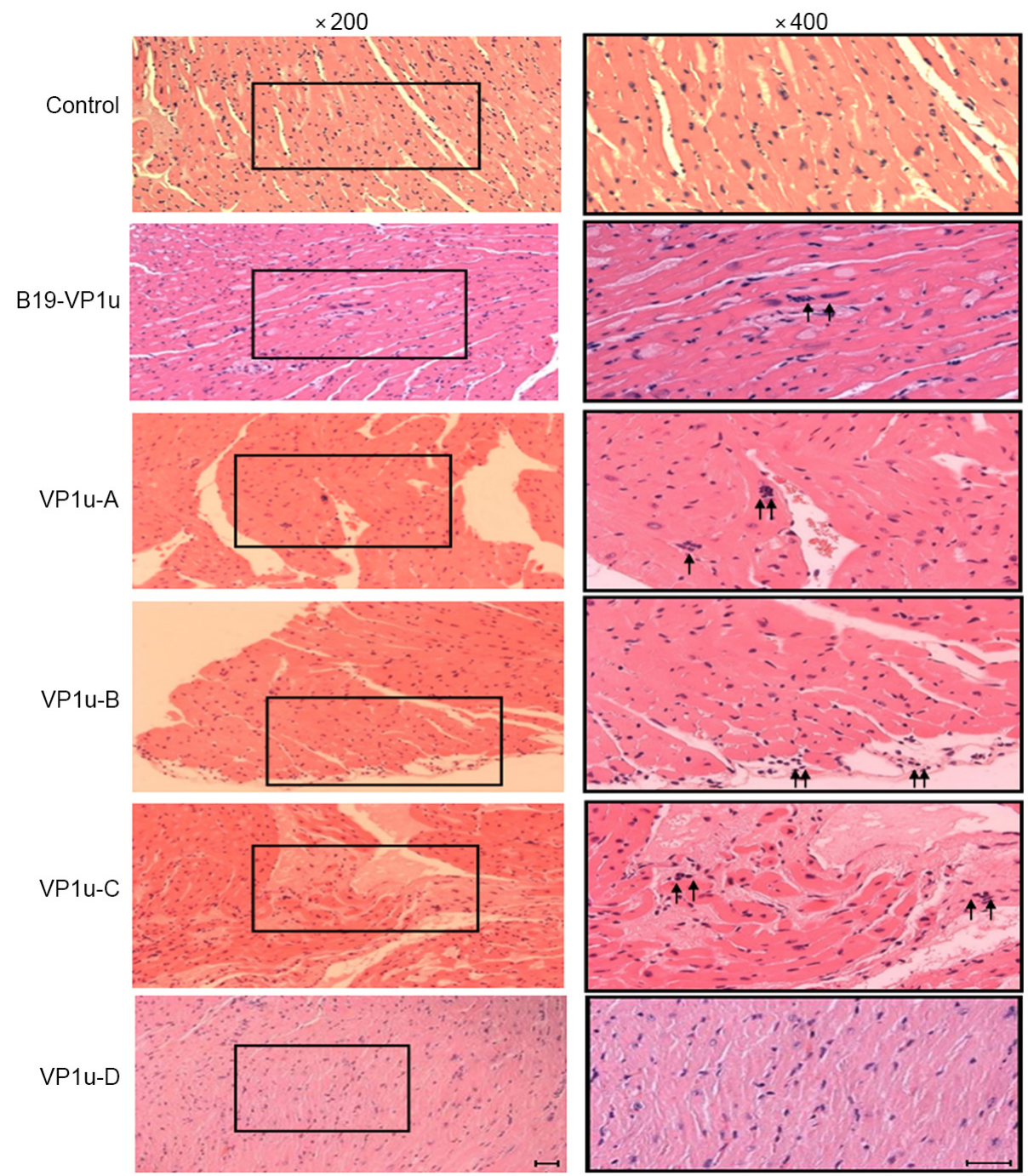

Figure 8. H\&E staining of the left ventricles in naïve mice treated with PBS (control), B19-VP1u, VP1u-A, VP1u-B, VP1u-C or VP1u-D. Lymphocyte infiltration was indicated by an arrow in the left ventricle tissues of naïve mice receiving different treatments (PBS, VP1u, B19-VP1u-A, B19-VP1u-B, B19-VP1u-C or B19-VP1u-D). The images in the left panel and right panel were magnified by x200 and x400, respectively. Scale bar, $10 \mu \mathrm{m}$. B19V, B19 virus; VP1u, VP1-unique region.

B19V-VP1u-D. These results are similar to previous findings, which revealed that B19V-VP1u could significantly increase cardiac injury $(11,25)$ and the first to show the differential effects of recombinant B19V-VP1u fragments on inducing the expression of cardiac injury markers.

MMPs, metal-bound enzymes that require zinc and calcium for their enzymatic activity and stability, are pivotal mediators involved in the remodeling of the extracellular matrix (33). Numerous studies have reported the essential roles of MMPs in organ development and subsequent tissue remodeling during inflammation and injury (34). MMPs, specifically MMP-9, also participate in the remodeling processes that occur following various cardiac injuries, such as myocarditis, atherosclerosis, heart failure, myocardial infarction and DCM (35). Indeed, MMP-9 has been shown to be involved in the inflammation and cardiac remodeling of Chagas disease, and the ratio of MMP-9/MMP-2 is considered as a potential biomarker in delineating the risk of cardiovascular injury (36).

ANP is expressed in the atria and ventricular myocardium, and its major physiological function is to reduce cardiac output, as well as to lower blood volume and systemic blood pressure (37). ANP is directly associated with increased stress caused by stretching, and is considered as a marker of congestive heart failure in both animal models and humans (38). ANP is known as a biochemical marker of cardiac hypertrophy, which ultimately leads to ventricular dilatation and heart failure (39). FABPs are expressed in various cells and tissues, such as cardiomyocytes, red skeletal muscle, kidney, hepatocytes, small intestine and adipocytes, and are associated with fatty acid metabolism (40). H-FABP is representative of its family in cardiac and red skeletal muscle, and is recognized as a biomarker of heart failure, chronic obstructive pulmonary disease and long-term postischemic prognosis $(41,42)$. Although CK-MB is not heart-specific, CK-MB is still used in the diagnosis of acute myocardial infarction $(43,44)$. To the best of our knowledge, the present study was the first to reveal that B19V-VP1u-A and B19V-VP1u-B increased ANP, H-FABP and $\mathrm{CK}-\mathrm{MB}$ expression levels in the left ventricles of naïve mice compared with the controls, whereas no differences in ANP, H-FABP and CK-MB expression levels were observed in 
A

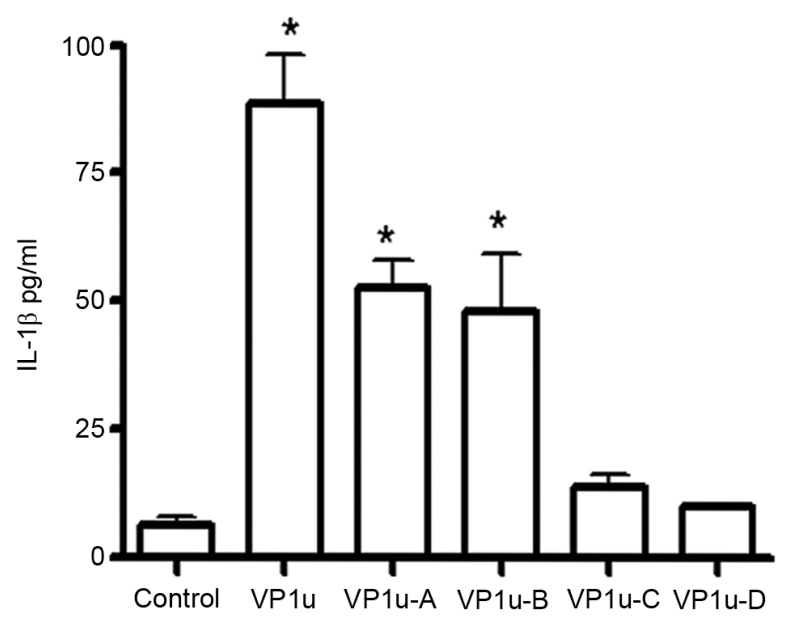

C

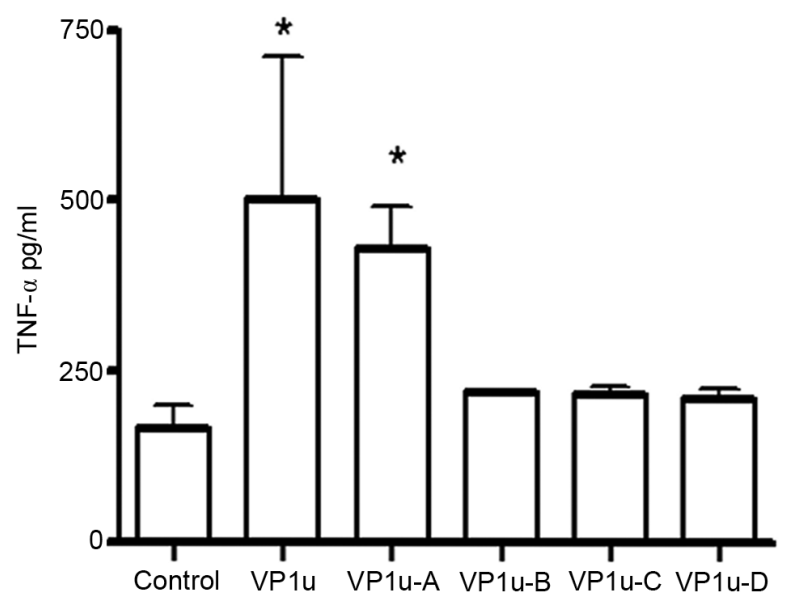

B

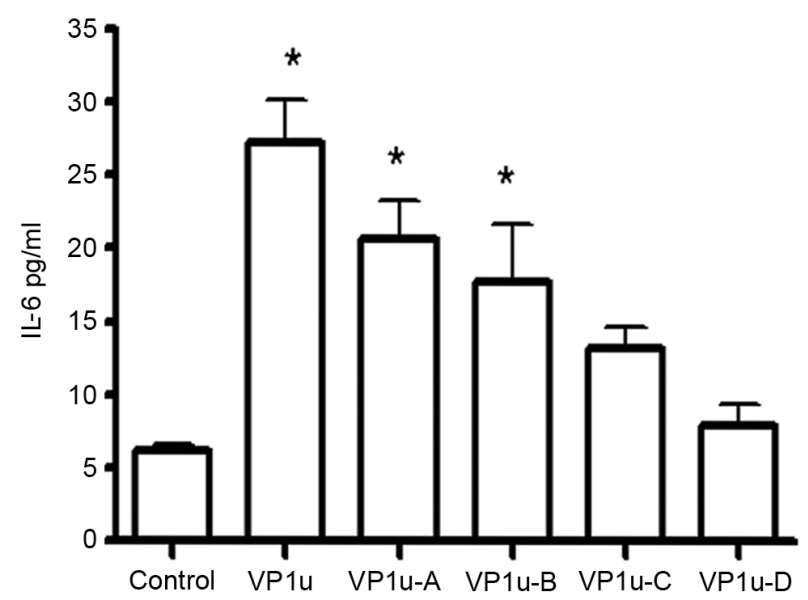

D

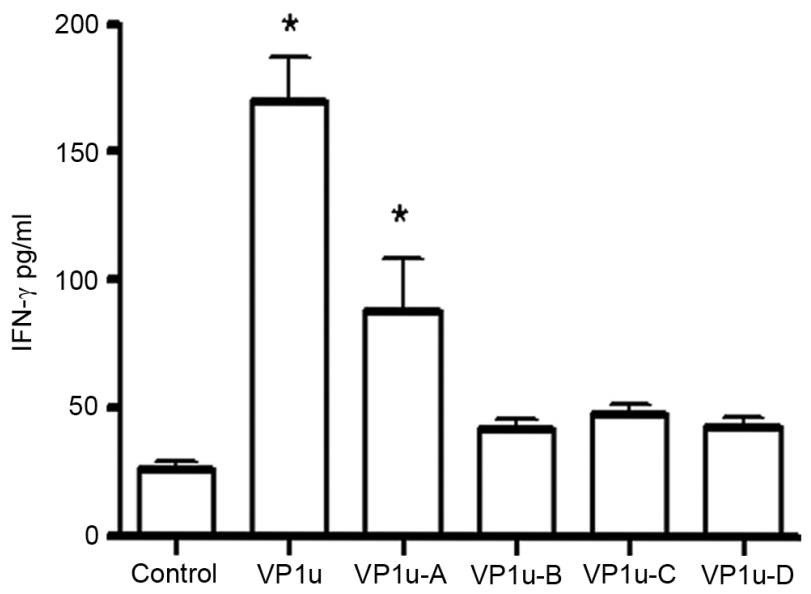

Figure 9. Levels of serum cytokines. The serum (A) IL-1 $\beta$, (B) IL-6, (C) TNF- $\alpha$ and (D) IFN- $\gamma$ levels in mice treated with B19V-VP1u, VP1u-A, VP1u-B, VP1u-C or VPlu-D were detected using Bio-Plex mouse cytokine assay kits. Data are presented as the mean \pm SD. Similar results were obtained in three-repeated experiments. " $\mathrm{P}<0.05$ vs. Control. $\mathrm{n}=4$ for each group. B19V, B19 virus; VP1u, VP1-unique region.

the mice treated with B19V-VP1u-C and B19V-VP1u-D. These findings suggest that the $\mathrm{N}$-terminal residues (residues 1-129) of B19V-VPlu exert a notable effect on the induction of the expression of various cardiac injury markers in the left ventricle.

Various cytokines serve essential roles in the pathogenesis of myocarditis and cardiomyopathy $(45,46)$. Elevated plasma TNF- $\alpha$, IL- 6 and IL-1 $\beta$ levels have been reported in patients suffering from acute myocardial infarction $(47,48)$. Evidence from both human and animal models has shown that proinflammatory cytokines, such as IL-1 $\beta$, IL-2, IL- 6 and TNF- $\alpha$, are involved in myocarditis, cardiac depression and heart failure $(49,50)$. The present study reported similar results, that B19V-VP1u, B19V-VP1u-A and B19V-VP1u-B increased the levels of IL-1 $\beta$ and IL- 6 in the left ventricle of naïve mice. Moreover, significantly increased levels of phosphorylation of P38 and ERK were also detected, which was consistent with previous findings showing that both ERK and P38 signaling are involved in the induction of IL-1 $\beta$ and IL-6 (51). These results demonstrated that the $129 \mathrm{~N}$-terminal amino acids of
B19V-VP1u (B19V-VP1u-A and B19V-VP1u-B) served key roles in inducing IL-1 $\beta$ and IL-6 levels via the ERK and P38 signaling pathways.

Previous studies have shown that the neutralizing epitopes of $\mathrm{B} 19 \mathrm{~V}$ are located within the N-terminal 100 amino acids of B19-VP1u, which contributes to B19V-induced immune responses $(52,53)$. The $\mathrm{N}$-terminal amino acids from residues 5-80 of B19-VP1u are essential for cellular binding and B19V uptake (54), which indicates an important role of the $\mathrm{N}$-terminal region of B19V-VP1u in immune regulation following B19V infection. However, little is known regarding the functional motifs in the C-terminal domain of B19V-VPlu. To the best of our knowledge, the present study was the first to show that IL-1 $\beta$ and IL-6 levels were significantly lower in the left ventricles of mice treated with B19V-VP1u-D (amino acids 196-227) compared with mice from the control group. This finding prompts a rational assumption that a negative regulatory motif on IL- $1 \beta$ and IL- 6 may be located within the C-terminal region of B19V-VP1u. However, further studies are required to verify the precise location of the motif and determine its function. 
There were certain limitations to the present study. Firstly, evidence has revealed that engagement of B19V-VP2 and globoside (P-antigen), and the following interaction of B19V-VP1 and $\alpha 5 \beta 1$ integrin are essential for B19 infection of erythroid cells and their progenitors (55). However, to the best of our knowledge, no study has investigated the potential receptor for B19V-VPlu and its truncated fragments on cardiac cells. Therefore, further research is required to verify the possible receptors on cardiac cells, as well as their downstream interactions. Second, the present study lacked the assessment of cardiac function using echocardiography or electrocardiography, which may provide further understanding of the physiological role of truncated B19V-VPlu fragments on cardiac injuries. Third, the underlying mechanism and related signaling molecules involved in truncated B19V-VP1u fragment-induced cardiac injury remain unknown, as do the mechanism via which B19V DNA entering cardiomyocytes. These issues require further investigations to elucidate the differential effects and mechanisms of truncated B19V-V1u fragments on cardiac injury.

In conclusion, the present study identified the differential effects of truncated B19V-VP1u fragments and its ability to induce an increase in the levels of cardiac inflammatory and injury markers in naïve mice. The $129 \mathrm{~N}$-terminal residues of B19V-VP1u, including B19V-VP1u-A and B19V-VP1u-B, predominantly induced the expression of inflammatory proteins, such as MMP-9, cardiac IL-1 $\beta$ and IL-6, and increased the serum levels of IL- $1 \beta$, IL- 6 , TNF- $\alpha$ and IFN- $\gamma$, as well as cardiac injury markers, such as ANP, H-FABP and CK-MB. Conversely, the C-terminal residues (amino acids 196-227) attenuated the levels of proinflammatory cytokines, including IL-1 $\beta$ and IL-6, but did not affect MMP-9 activity or the induction of cardiac injury markers. Taken together, these findings may provide further information and improve the current understanding of the regulatory roles of B19V-VPlu in inducing cardiac injury.

\section{Acknowledgements}

Not applicable.

\section{Funding}

This study was supported by Chung Shan Medical University and Chi-Mei Medical Center cooperative project CSMU-CMMC-108-01 (grant no. CMCSMU10801) and in part by the Ministry of Science and Technology (grant nos. MOST 106-2314-B040-023, 107-2314-B040-004 and 109-2314-B040-021), Taiwan. The funders had no role in study design, data collection and analysis, decision to publish, or preparation of the manuscript.

\section{Availability of data and materials}

The datasets used and/or analyzed during the current study are available from the corresponding author on reasonable request.

\section{Authors' contributions}

$\mathrm{KCH}$ was involved in the study conception and design, and analysis of data. ZYH and JLY performed experiments and analysis of data. TCH was involved in the study conception and design, drafting and revising of the manuscript, performing experiments, analysis of data, and study supervision. BST was involved in the study conception and design, drafting and revising of the manuscript, and analysis of data. TCH and BST confirm the authenticity of all the raw data. All authors read and approved the final manuscript.

\section{Ethics approval and consent to participate}

Animal experiments were performed in accordance with the principles of replacement, refinement and reduction and were approved by the Institutional Animal Care and Use Committee (IACUC) of Chung Shan Medical University, Taichung (approval no. 1676; approved in December 2015).

\section{Patient consent for publication}

Not applicable.

\section{Competing interests}

The authors declare that they have no competing interests.

\section{References}

1. Kelly HA, Siebert D, Hammond R, Leydon J, Kiely P and Maskill W: The age-specific prevalence of human parvovirus immunity in Victoria, Australia compared with other parts of the world. Epidemiol Infect 124: 449-457, 2000.

2. Cotmore SF, McKie VC, Anderson LJ, Astell CR and Tattersall P: Identification of the major structural and nonstructural proteins encoded by human parvovirus B19 and mapping of their genes by procaryotic expression of isolated genomic fragments. J Virol 60: 548-557, 1986.

3. Nüesch JPF, Corbau R, Tattersall P and Rommelaere J: Biochemical activities of minute virus of mice nonstructural protein NS1 Are modulated in vitro by the phosphorylation state of the polypeptide. J Virol 72: 8002-8012, 1998.

4. Tewary SK, Zhao H, Deng X, Qiu J and Tang L: The human parvovirus B19 non-structural protein $1 \mathrm{~N}$-terminal domain specifically binds to the origin of replication in the viral DNA. Virology 449: 297-303, 2014.

5. Moffatt S, Yaegashi N, Tada K, Tanaka N and Sugamura K: Human parvovirus B19 nonstructural (NS1) protein induces apoptosis in erythroid lineage cells. J Virol 72: 3018-3028, 1998.

6. Fu Y, Ishii KK, Munakata Y, Saitoh T, Kaku M and Sasaki T: Regulation of tumor necrosis factor alpha promoter by human parvovirus B19 NS1 through activation of AP-1 and AP-2. J Virol 76: 5395-5403, 2002.

7. Weigel-Kelley KA, Yoder MC and Srivastava A: Recombinant human parvovirus B19 vectors: Erythrocyte P antigen is necessary but not sufficient for successful transduction of human hematopoietic cells. J Virol 75: 4110-4116, 2001.

8. Kurtzman GJ, Cohen BJ, Field AM, Oseas R, Blaese RM and Young NS: Immune response to B19 parvovirus and an antibody defect in persistent viral infection. J Clin Invest 84: 1114-1123, 1989.

9. Anderson S, Momoeda M, Kawase M, Kajigaya S and Young NS: Peptides derived from the unique region of B19 parvovirus minor capsid protein elicit neutralizing antibodies in rabbits. Virology 206: 626-632, 1995.

10. Mahrholdt H, Wagner A, Deluigi CC, Kispert E, Hager S, Meinhardt G, Vogelsberg H, Fritz P, Dippon J, Bock CT, et al: Presentation, patterns of myocardial damage, and clinical course of viral myocarditis. Circulation 114: 1581-1590, 2006.

11. Bogomolovas J, Šimoliūnas E, Rinkūnaitè I, Smalinskaitè L, Podkopajev A, Bironaitė D, Weis CA, Marx A, Bukelskienė V, Gretz N, et al: A novel murine model of parvovirus associated dilated cardiomyopathy induced by immunization with VP1-unique region of parvovirus B19. Biomed Res Int 2016: 1627184, 2016. 
12. Hatakka A, Klein J, He R, Piper J, Tam E and Walkty A: Acute hepatitis as a manifestation of parvovirus B19 infection. J Clin Microbiol 49: 3422-3424, 2011.

13. Komatsuda A, Ohtani H, Nimura T, Yamaguchi A, Wakui H, Imai $\mathrm{H}$ and Miura AB: Endocapillary proliferative glomerulonephritis in a patient with parvovirus B19 infection. Am J Kidney Dis 36: 851-854, 2000.

14. Ferrari SM, Fallahi P, Antonelli A and Benvenga S: Environmental issues in thyroid diseases. Front Endocrinol (Lausanne) 8: 50, 2017

15. Bozzola E, Krzysztofiak A and Cortis E: Neurological impairment and arthritis in an immunocompetent child with human parvovirus B19 chronic infection. Infez Med 18: 187-190, 2010.

16. Hobbs JA and Adamson-Small LA: Parvovirus and thyroid cancer. Semin Oncol 42: 304-308, 2015.

17. Page C, François C, Goëb V and Duverlie G: Human parvovirus B19 and autoimmune diseases. Review of the literature and pathophysiological hypotheses. J Clin Virol 72: 69-74, 2015.

18. Pankuweit S, Moll R, Baandrup U, Portig I, Hufnagel G and Maisch B: Prevalence of the parvovirus B19 genome in endomyocardial biopsy specimens. Hum Pathol 34: 497-503, 2003.

19. Pankuweit S, Lamparter S, Schoppet $M$ and Maisch B Parvovirus B19 genome in endomyocardial biopsy specimen. Circulation 109: e179, 2004.

20. Tschöpe C, Bock CT, Kasner M, Noutsias M, Westermann D, Schwimmbeck PL, Pauschinger M, Poller WC, Kühl U, Kandolf R and Schultheiss HP: High prevalence of cardiac parvovirus B19 infection in patients with isolated left ventricular diastolic dysfunction. Circulation 111: 879, 2005.

21. Kühl U, Lassner D, Pauschinger M, Gross UM, Seeberg B, Noutsias M, Poller W and Schultheiss HP: Prevalence of erythrovirus genotypes in the myocardium of patients with dilated cardiomyopathy. J Med Virol 80: 1243-1251, 2008.

22. Bültmann BD, Klingel K, Sotlar K, Bock CT, Baba HA, Sauter M and Kandolf R: Fatal parvovirus B19-associated myocarditis clinically mimicking ischemic heart disease: An endothelial cell-mediated disease. Hum Pathol 34: 92-95, 2003.

23. Duechting A, Tschöpe C, Kaiser H, Lamkemeyer T, Tanaka N, Aberle S, Lang F, Torresi J, Kandolf R and Bock CT: Human parvovirus B19 NS1 protein modulates inflammatory signaling by activation of STAT3/PIAS3 in human endothelial cells. J Virol 82: 7942-7952, 2008.

24. Hii HP, Chiu CC, Lin DW, Shi YF, Hsu TC and Tzang BS: Selective activation of inflammation factors by human parvovirus B19 and human bocavirus VP1 unique region on H9c2 cardiomyocyte. Mol Med Rep 18: 4072-4078, 2018.

25. Nie X, Zhang G, Xu D, Sun X, Li Z, Li X, Zhang X, He F and $\mathrm{Li}$ Y: The VP1-unique region of parvovirus B19 induces myocardial injury in mice. Scand J Infect Dis 42: 121-128, 2010.

26. Tzang BS, Lin TM, Tsai CC, Hsu JD, Yang LC and Hsu TC: Increased cardiac injury in NZB/W F1 mice received antibody against human parvovirus B19 VP1 unique region protein. Mol Immunol 48: 1518-1524, 2011.

27. Chen DY, Chen YM, Tzang BS, Lan JL and Hsu TC: Th17-related cytokines in systemic lupus erythematosus patients with dilated cardiomyopathies: A possible linkage to parvovirus B19 infection. PLoS One 9: e113889, 2014.

28. Tsai CC, Chiu CC, Hsu JD, Hsu HS, Tzang BS and Hsu TC: Human parvovirus B19 NS1 protein aggravates liver injury in NZB/W F1 mice. PLoS One 8: e59724, 2013

29. Lin CY, Chiu CC, Cheng J, Lin CY, Shi YF, Tsai CC, Tzang BS and Hsu TC: Antigenicity analysis of human parvovirus B19-VP1u protein in the induction of anti-phospholipid syndrome. Virulence 9: 208-216, 2018.

30. Nakashima I, Ota F, Kobayashi T, Kato O and Kato N: The effect of antigen doses and time intervals between antigen injections on secondary, tertiary and quaternary antibody responses. Establishment of hyperimmunization with bovine serum albumin in mice treated with capsular polysaccharide of Klebsiella pneumoniae. Immunology 26: 443-454, 1974.

31. Castiglione F, Mantile F, De Berardinis P and Prisco A: How the interval between prime and boost injection affects the immune response in a computational model of the immune system. Comput Math Methods Med 2012: 842329, 2012.

32. Hsu TC, Chiu CC, Chang SC, Chan HC, Shi YF, Chen TY and Tzang BS: Human parvovirus B19 VP1u Protein as inflammatory mediators induces liver injury in naïve mice. Virulence 7 : 110-118, 2016.
33. Liu P, Sun M and Sader S: Matrix metalloproteinases in cardiovascular disease. Can J Cardiol 22 (Suppl B): 25B-30B, 2006

34. Liu J and Khalil RA: Matrix metalloproteinase inhibitors as investigational and therapeutic tools in unrestrained tissue remodeling and pathological disorders. Prog Mol Biol Transl Sci 148: 355-420, 2017.

35. Kampoli AM, Tousoulis D, Papageorgiou N, Antoniades C, Androulakis E, Tsiamis E, Latsios G and Stefanadis C: Matrix metalloproteinases in acute coronary syndromes: Current perspectives. Curr Top Med Chem 12: 1192-1205, 2012.

36. Fares RC, Gomes Jde A, Garzoni LR, Waghabi MC, Saraiva RM, Medeiros NI, Oliveira-Prado R, Sangenis LH, Chambela Mda C, de Araújo FF, et al: Matrix metalloproteinases 2 and 9 are differentially expressed in patients with indeterminate and cardiac clinical forms of Chagas disease. Infect Immun 81: 3600-3608, 2013

37. Edwards BS, Zimmerman RS and Burnett JC Jr: Atrial natriuretic factor: Physiologic actions and implications in congestive heart failure. Cardiovasc Drugs Ther 1: 89-100, 1987.

38. Michel JB, Arnal JF and Corvol P: Atrial natriuretic factor as a marker in congestive heart failure. Horm Res 34: 166-168, 1990.

39. Rohini A, Agrawal N, Koyani CN and Singh R: Molecular targets and regulators of cardiac hypertrophy. Pharmacol Res 61: 269-280, 2010

40. Vork MM, Glatz JF and van Der Vusse GJ: On the mechanism of long chain fatty acid transport in cardiomyocytes as facilitated by cytoplasmic fatty acid-binding protein. J Theor Biol 160: 207-222, 1993

41. Crisman TS, Claffey KP, Saouaf R, Hanspal J and Brecher P: Measurement of rat heart fatty acid binding protein by ELISA. Tissue distribution, developmental changes and subcellular distribution. J Mol Cell Cardiol 19: 423-431, 1987.

42. Sato Y, Kita T, Takatsu Y and Kimura T: Biochemical markers of myocyte injury in heart failure. Heart 90: 1110-1113, 2004.

43. Mair J: Progress in myocardial damage detection: New biochemical markers for clinicians. Crit Rev Clin Lab Sci 34: $1-66,1997$.

44. Aydin S, Ugur K, Aydin S, Sahin İ and Yardim M: Biomarkers in acute myocardial infarction: Current perspectives. Vasc Health Risk Manag 15: 1-10, 2019.

45. Levine B, Kalman J, Mayer L, Fillit HM and Packer M: Elevated circulating levels of tumor necrosis factor in severe chronic heart failure. N Engl J Med 323: 236-241, 1990.

46. Matsumori A: Molecular and immune mechanisms in the pathogenesis of cardiomyopathy-role of viruses, cytokines, and nitric oxide. Jpn Circ J 61: 275-291, 1997.

47. Miyao Y, Yasue H, Ogawa H, Misumi I, Masuda T, Sakamoto T and Morita E: Elevated plasma interleukin-6 levels in patients with acute myocardial infarction. Am Heart J 126: 1299-1304, 1993.

48. Neumann FJ, Ott I, Gawaz M, Richardt G, Holzapfel H, Jochum M and Schömig A: Cardiac release of cytokines and inflammatory responses in acute myocardial infarction. Circulation 92: 748-755, 1995.

49. Dinarello CA: The biological properties of interleukin-1. Eur Cytokine Netw 5: 517-531, 1994.

50. Blum A and Miller H: Role of cytokines in heart failure. Am Heart J 35: 181-186, 1998.

51. Carter AB, Monick MM and Hunninghake GW: Both Erk and p38 kinases are necessary for cytokine gene transcription. Am J Respir Cell Mol Biol 20: 751-758, 1999.

52. Saikawa T, Anderson S, Momoeda M, Kajigaya S and Young NS: Neutralizing linear epitopes of B19 parvovirus cluster in the VP1 unique and VP1-VP2 junction regions. J Virol 67: 3004-3009, 1993.

53. Zuffi E, Manaresi E, Gallinella G, Gentilomi GA, Venturoli S, Zerbini $\mathrm{M}$ and Musiani M: Identification of an immunodominant peptide in the parvovirus B19 VP1 unique region able to elicit a long-lasting immune response in humans. Viral Immunol 14: 151-158, 2001.

54. Leisi R, Di Tommaso C, Kempf C and Ros C: The receptor-binding domain in the VPlu region of parvovirus B19. Viruses 8: 61, 2016.

55. Qiu J, Söderlund-Venermo M and Young NS: Human parvoviruses. Clin Microbiol Rev 30: 43-113, 2017.

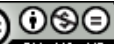

This work is licensed under a Creative Commons Attribution-NonCommercial-NoDerivatives 4.0 International (CC BY-NC-ND 4.0) License. 